\title{
PARABOLIC EQUATIONS WITH VARIABLY PARTIALLY VMO COEFFICIENTS
}

\author{
H. DONG
}

\begin{abstract}
The $W_{p}^{1,2}$-solvability of second-order parabolic equations in nondivergence form in the whole space is proved for $p \in(1, \infty)$. The leading coefficients are assumed to be measurable in one spatial direction and have vanishing mean oscillation (VMO) in the orthogonal directions and the time variable in each small parabolic cylinder with direction allowed to depend on the cylinder. This extends a recent result by Krylov for elliptic equations. The novelty in the current paper is that the restriction $p>2$ is removed.
\end{abstract}

\section{§1. INTRODUCTION}

We consider the $W_{p}^{1,2}$-solvability of parabolic equations in nondivergence form:

$$
P u-\lambda u=f,
$$

where $\lambda \geq 0$ is a constant, $f \in L_{p}$, and

$$
P u=-u_{t}+a^{i j} D_{i j} u+b^{j} D_{j} u+c u
$$

is a uniformly nondegenerate parabolic operator with bounded coefficients.

The $L_{p}$-theory of second-order parabolic and elliptic equations has been studied extensively under various regularity assumptions on the coefficients. For equations with uniformly continuous leading coefficients, the solvability has been known for a long time; see, for example, Agmon, Douglis, and Nirenberg [3] and Ladyzhenskaya, Solonnikov and Ural'tseva [20]. With VMO coefficients, the solvability theorems of nondivergence form equations were established in the early 1990s by Chiarenza, Frasca, and Longo [5, 6] and by Bramanti and Cerutti [4]. The main technical tool in these papers was the theory of singular integrals, in particular, certain estimates from the Calderón-Zygmund theorem and the Coifman-Rochberg-Weiss commutator theorem. For divergence form equations with VMO coefficients, we refer the reader to Di Fazio [10, Byun and Wang 2], Acerbi and Mingione [1, and the references therein.

On the contrary, the theory of elliptic and parabolic equations with partially VMO coefficients was quite new and originated by Kim and Krylov in [14] and [15]. In [14, the authors established the $W_{p}^{2}$-solvability of elliptic equations in nondivergence form under the assumption that the coefficients $a^{i j}$ are measurable with respect to $x^{1}$ and VMO with respect to the remaining variables. This result was extended to parabolic equations by the same authors in [15]. The arguments in [14] and [15] were based on the method previously developed by Krylov in [17] and [18, where the author gave a unified approach to studying the $L_{p}$-solvability of both divergence and nondivergence form parabolic equations with leading coefficients measurable in the time variable and

2010 Mathematics Subject Classification. Primary 35K15, 35R05.

Key words and phrases. Second-order equations, vanishing mean oscillation, partially VMO coefficients, Sobolev spaces.

Partially supported by NSF Grant DMS-0635607 from IAS and NSF Grant DMS-0800129. 
VMO in the spatial variables. Unlike the arguments in [5, 6, 4, the proofs in [17] and [18 rely mainly on pointwise estimates of sharp functions of spatial derivatives of solutions 1 We also mention that the results in [15, 17, 18] were improved by Kim in [11, 12, 13], where most leading coefficients are measurable in the time variable and one spatial variable, and VMO in the other variables. In Dong and Krylov [9, the $W_{p}^{2}$-solvability was obtained for equations with leading coefficients measurable in two spatial variables and VMO in the others, when $p>2$ is sufficiently close to 2 .

The $W_{p}^{1}$ and $\mathcal{H}_{p}^{1}$-solvability of elliptic and parabolic equations in divergence form with partially BMO coefficients was obtained in the recent papers [8] and [7.

The result in [14] was generalized very recently by Krylov [19] to nondivergence form elliptic equations with variably partially VMO coefficients. More precisely, the leading coefficients were assumed to be measurable in one direction and VMO in the orthogonal directions in each small ball with direction depending on the ball. Roughly speaking, the main idea in [19] was to use in a localized way a pointwise sharp function estimate of a portion of the Hessian $D^{2} u$, proved in [14, and apply a generalized version of the Fefferman-Stein theorem on sharp functions. Rather than looking for as weak regularity assumptions on coefficients as possible, we emphasize that one of the main points of these papers was to put forth a new technique, which turned out to be more powerful than singular integrals in several occasions.

As was pointed out in [19, a restriction of the result there (and also in papers mentioned above regarding nondivergence equations with partially VMO coefficients) was that $p$ must be greater than 2. This restriction is due to the following reason. The sharp function estimate in [14 was deduced from the $W_{2}^{2}$-solvability of equations with $a^{i j}$ depending only on $x^{1}$, which was obtained by using the method of the Fourier transformation. In its turn, the right-hand side of the estimate contains maximal functions of $q$ th power of $D^{2} u$ for some $q>2$. Therefore, to apply the Fefferman-Stein theorem and the Hardy-Littlewood maximal function theorem one requires $p>q>2$.

It is natural to ask if we still have the $W_{p}^{2}$-solvability under the same assumptions of the coefficients when $p$ goes below 2. In this paper, we give a positive answer to this question. We obtain the $W_{p}^{2}$-solvability of nondivergence form elliptic equations for any $p \in(1, \infty)$ (stated in Theorem 2.5), under the same assumptions as in [19, i.e., the leading coefficients are variably partially VMO. In fact, we shall establish the corresponding $W_{p}^{1,2}$-solvability of parabolic equations, from which the result of elliptic equations follows in a standard way. Here the leading coefficients of parabolic equations are assumed to be measurable in one spatial direction and have vanishing mean oscillation in the orthogonal directions and the time variable in each small parabolic cylinder, with the direction allowed to depend on the cylinder. For the precise statement of this result, see Theorem 2.2. This theorem generalizes the result in [15] as well. Additionally, in the spirit of [18, we also obtain the solvability of parabolic equations in the mixed-norm Sobolev spaces $W_{q, p}^{1,2}$ for $q \geq p$ under the same assumptions (see Theorem 5.1).

The proofs follow the approach in [19] with two new ideas outlined below to get around the obstacles. First, in order to go below 2, we shall establish for any $p \in(1, \infty)$ the $W_{p}^{1,2}$-solvability of nondivergence form parabolic equations with $a^{i j}$ depending only on $x^{1}$ (stated in Theorem 3.4). For this purpose, we observe that with this structure the equation can be rewritten into a divergence form after a suitable change of variables. This enables us to apply a result recently proved in [7. for divergence form parabolic equations with partially VMO coefficients. Second, to get a sharp function estimate we need to bound the Hölder norms of a portion of $D^{2} u$ when $u$ satisfies the homogeneous

\footnotetext{
${ }^{1}$ See also 22 for a straightforward treatment of nondivergence form elliptic and parabolic equations in the Morrey space without using any singular integrals.
} 
equation (see Theorem 3.7). The proof of a similar estimate in Lemma 4.8 in [14, which was used crucially in [19], is only for $p=2$. To this end, we use a novel bootstrap argument with the aid of Theorem 3.4 and an embedding type estimate. We combine Theorems 3.4 and 3.7 to prove Theorem 2.2 by applying the aforementioned generalized Fefferman-Stein theorem obtained in [19].

A possible application of the results is the solvability of elliptic and parabolic equations in domains, with the Dirichlet or Neumann boundary condition; see [14] and [8] for various discussions. We believe that the results in this paper enable us to obtain the solvability of equations with partially VMO coefficients for which the measurable direction is close to the normal direction near the boundary. We do not intend to pursue this in the current paper.

A brief outline of the paper: in the next section, we introduce the notation and state the main results. $₫ 3$ contains a few preliminary estimates, including Theorems 3.4 and 3.7, which are the main ingredients of the proof. We finish the proof of the $W_{p}^{1,2}$-solvability in $₫ 4$ by combining the results of $₫ 3$. Finally, we state and prove the $W_{q, p}^{1,2}$-solvability of parabolic equations in the last section.

\section{§2. Notation And MAin RESUlts}

We begin the section by introducing some notation. Let $d \geq 2$ be an integer. A typical point in $\mathbb{R}^{d}$ is denoted by $x=\left(x^{1}, x^{2}, \ldots, x^{d}\right)=\left(x^{1}, x^{\prime}\right)$. By $D u=\left(D_{i} u\right)$ and $D^{2} u=$ $\left(D_{i j} u\right)$ we mean the gradient and the Hessian matrix of $u$. On many occasions we need to take these objects relative to only part of variables. We also use the following notation:

$$
D_{x^{\prime}} u=u_{x^{\prime}}, \quad D_{x^{1} x^{\prime}} u=u_{x^{1} x^{\prime}}, \quad D_{x x^{\prime}} u=u_{x x^{\prime}} .
$$

Throughout the paper, we always assume that $1<p, q<\infty$ unless explicitly specified otherwise. Writing $N(d, p, \ldots)$, we mean that $N$ is a constant depending only on the prescribed quantities $d, p, \ldots$. For a (matrix-valued) function $f(t, x)$ in $\mathbb{R}^{d+1}$, we set

$$
(f)_{\mathcal{D}}=\frac{1}{|\mathcal{D}|} \int_{\mathcal{D}} f(t, x) d x d t=f_{\mathcal{D}} f(t, x) d x d t
$$

where $\mathcal{D}$ is an open subset in $\mathbb{R}^{d+1}$ and $|\mathcal{D}|$ is the $(d+1)$-dimensional Lebesgue measure of $\mathcal{D}$. For $-\infty \leq S<T \leq \infty$ and $\Omega \subset \mathbb{R}^{d}$, we set

$$
L_{q, p}((S, T) \times \Omega)=L_{q}\left((S, T), L_{p}(\Omega)\right),
$$

i.e., $f(t, x) \in L_{q, p}((S, T) \times \Omega)$ if

$$
\|f\|_{L_{q, p}((S, T) \times \Omega)}=\left(\int_{S}^{T}\left(\int_{\Omega}|f(t, x)|^{p} d x\right)^{q / p} d t\right)^{1 / q}<\infty .
$$

Denote

$$
\begin{aligned}
L_{p}((S, T) \times \Omega) & =L_{p, p}((S, T) \times \Omega), \\
W_{q, p}^{1,2}((S, T) \times \Omega) & =\left\{u: u, u_{t}, D u, D^{2} u \in L_{q, p}((S, T) \times \Omega)\right\}, \\
W_{p}^{1,2}((S, T) \times \Omega) & =W_{p, p}^{1,2}((S, T) \times \Omega) .
\end{aligned}
$$

We also use the abbreviations $L_{p}=L_{p}\left(\mathbb{R}^{d+1}\right), W_{p}^{1,2}=W_{p}^{1,2}\left(\mathbb{R}^{d+1}\right)$, etc. For $\alpha \in(0,1]$, we denote the $C^{\alpha / 2, \alpha}$-seminorm by

$$
[u]_{C^{\alpha / 2, \alpha}((S, T) \times \Omega)}:=\sup _{\substack{(t, x),(s, y) \in(S, T) \times \Omega \\(t, x) \neq(s, y)}} \frac{|u(t, x)-u(s, y)|}{|t-s|^{\alpha / 2}+|x-y|^{\alpha}},
$$


and the $C^{\alpha / 2, \alpha}$-norm by

$$
\|u\|_{C^{\alpha / 2, \alpha}((S, T) \times \Omega)}:=[u]_{C^{\alpha / 2, \alpha}((S, T) \times \Omega)}+\|u\|_{L_{\infty}((S, T) \times \Omega)} .
$$

For any $T \in(-\infty, \infty]$ and $\Omega \subset \mathbb{R}^{d}$, we denote

$$
\mathbb{R}_{T}=(-\infty, T), \quad \mathbb{R}_{T}^{d+1}=\mathbb{R}_{T} \times \mathbb{R}^{d} .
$$

Let

$$
B_{r}(x)=\left\{y \in \mathbb{R}^{d}:|x-y|<r\right\}, \quad Q_{r}(t, x)=\left(t-r^{2}, t\right) \times B_{r}(x) .
$$

Set $B_{r}=B_{r}(0), Q_{r}=Q_{r}(0,0)$, and let $\left|B_{r}\right|$ and $\left|Q_{r}\right|$ be the volume of $B_{r}$ and $Q_{r}$ respectively. Let $\mathcal{Q}=\left\{Q_{r}(t, x):(t, x) \in \mathbb{R}^{d+1}, r \in(0, \infty)\right\}$. For a function $g$ defined on $\mathbb{R}^{d+1}$, we denote its (parabolic) maximal and sharp function, respectively, by

$$
\begin{aligned}
\mathbb{M} g(t, x) & =\sup _{Q \in \mathcal{Q}:(t, x) \in Q} f_{Q}|g(s, y)| d y d s, \\
g^{\#}(t, x) & =\sup _{Q \in \mathcal{Q}:(t, x) \in Q} f_{Q}\left|g(s, y)-(g)_{Q}\right| d y d s .
\end{aligned}
$$

Next we state our assumptions on the coefficients precisely. We assume that all the coefficients are bounded and measurable, and the $a^{j k}$ are symmetric uniformly elliptic, i.e.,

$$
\begin{aligned}
\left|b^{j}\right| & \leq K, \quad|c| \leq K, \\
\delta|\xi|^{2} & \leq a^{j k} \xi^{j} \xi^{k} \leq \delta^{-1}|\xi|^{2} .
\end{aligned}
$$

Denote by $\mathcal{A}$ the set of symmetric $(d \times d)$-matrix-valued measurable functions $\bar{a}=$ $\left(\bar{a}^{i j}\left(y^{1}\right)\right)$ of one spatial variable such that (2.1) holds with $\bar{a}$ in place of $a$.

Let $\Psi$ be the set of $C^{1,1}$-diffeomorphisms $\psi: \mathbb{R}^{d} \rightarrow \mathbb{R}^{d}$ such that the mappings $\psi$ and $\phi=\psi^{-1}$ satisfy

$$
|D \psi|+\left|D^{2} \psi\right| \leq \delta^{-1}, \quad|D \phi|+\left|D^{2} \phi\right| \leq \delta^{-1} .
$$

Assumption $2.1(\gamma)$. There exists a positive constant $R_{0} \in(0,1]$ such that, for any parabolic cylinder $Q$ of radius less than $R_{0}$, one can find $\bar{a} \in \mathcal{A}$ and $\psi=\left(\psi^{1}, \ldots, \psi^{d}\right) \in \Psi$ such that

$$
\int_{Q}\left|a(t, x)-\bar{a}\left(\psi^{1}(x)\right)\right| d x d t \leq \gamma|Q|
$$

Next we state the main result of the paper.

Theorem 2.2. For any $p \in(1, \infty)$, there exists $\gamma=\gamma(d, \delta, p)>0$ such that, under Assumption $2.1(\gamma)$, for any $T \in(-\infty,+\infty]$ the following holds.

i) For any $u \in W_{p}^{1,2}\left(\mathbb{R}_{T}^{d+1}\right)$,

$\lambda\|u\|_{L_{p}\left(\mathbb{R}_{T}^{d+1}\right)}+\sqrt{\lambda}\|D u\|_{L_{p}\left(\mathbb{R}_{T}^{d+1}\right)}+\left\|D^{2} u\right\|_{L_{p}\left(\mathbb{R}_{T}^{d+1}\right)}+\left\|u_{t}\right\|_{L_{p}\left(\mathbb{R}_{T}^{d+1}\right)} \leq N\|P u-\lambda u\|_{L_{p}\left(\mathbb{R}_{T}^{d+1}\right)}$, provided that $\lambda \geq \lambda_{0}$, where $\lambda_{0} \geq 0$ and $N$ depend only on $d, \delta, p, K$, and $R_{0}$.

ii) For any $\lambda>\lambda_{0}$ and $f \in L_{p}\left(\mathbb{R}_{T}^{d+1}\right)$, there exists a unique solution $u \in W_{p}^{1,2}\left(\mathbb{R}_{T}^{d+1}\right)$ of equation (1.1) in $\mathbb{R}_{T}^{d+1}$.

Theorem 2.2 yields the following solvability result of the initial value problem for parabolic equations.

Theorem 2.3. For any $p \in(1, \infty)$, there exists a $\gamma=\gamma(d, \delta, p)>0$ such that under Assumption $2.1(\gamma)$ for any $T \in(0, \infty)$ the following is true. For any $f \in L_{p}\left((0, T) \times \mathbb{R}^{d}\right)$, there exists a unique solution $u \in W_{p}^{1,2}\left((0, T) \times \mathbb{R}^{d}\right)$ of the initial value problem

$$
P u=f \text { in }(0, T) \times \mathbb{R}^{d}, \quad u(0, \cdot)=0 .
$$


Moreover, we have

$$
\|u\|_{W_{p}^{1,2}\left((0, T) \times \mathbb{R}^{d}\right)} \leq N\|f\|_{L_{p}\left((0, T) \times \mathbb{R}^{d}\right)},
$$

where $N$ depends only on $d, \delta, p, K, T$, and $R_{0}$.

Indeed, by considering $v:=u e^{-\left(\lambda_{0}+1\right) t}$ instead of $u$, the operator $P$ becomes $P-$ $\left(\lambda_{0}+1\right) I$. Now we extend $f$ to be zero for $t<0$ and solve the equation for $v$ in $\mathbb{R}_{T}^{d+1}$ using Theorem 2.2. By uniqueness of solutions in $W_{p}^{1,2}\left(\mathbb{R}_{0}^{d+1}\right)$, automatically $v=0$ when $t \leq 0$. Thus, $u=v e^{\left(\lambda_{0}+1\right) t}$ solves the original initial value problem and the estimate also follows. The uniqueness of $u$ is equivalent to the uniqueness of $v$. We extend $v$ to be zero for $t<0$. Then $v$ satisfies $P v-\left(\lambda_{0}+1\right) v=\chi_{t>0} e^{-\left(\lambda_{0}+1\right) t} f$ in $\mathbb{R}_{T}^{d+1}$, and so the uniqueness follows from Theorem 2.2 .

In the case where all the coefficients are time-independent, we also consider the $W_{p}^{2}$ solvability of elliptic equations in nondivergence form:

$$
L u-\lambda u=f,
$$

where

$$
L u=a^{j k} D_{j k} u+b^{j} D_{j} u+c u .
$$

Assumption $2.4(\gamma)$. There exists a positive constant $R_{0} \in(0,1]$ such that for any ball $B$ of radius less than $R_{0}$ one can find $\bar{a} \in \mathcal{A}$ and $\psi=\left(\psi_{1}, \ldots, \psi_{d}\right) \in \Psi$ such that

$$
\int_{B}\left|a(x)-\bar{a}\left(\psi^{1}(x)\right)\right| d x \leq \gamma|B| .
$$

The following $W_{p}^{2}$-solvability theorem for elliptic equations is an immediate corollary to Theorem 2.2, which generalizes Theorem 1.4 of [19] by dropping the condition $p>2$.

Theorem 2.5. For any $p \in(1, \infty)$, there exists $\gamma=\gamma(d, \delta, p)>0$ such that under Assumption $2.4(\gamma)$ the following holds true.

i) For any $u \in W_{p}^{2}\left(\mathbb{R}^{d}\right)$,

$$
\lambda\|u\|_{L_{p}\left(\mathbb{R}^{d}\right)}+\sqrt{\lambda}\|D u\|_{L_{p}\left(\mathbb{R}^{d}\right)}+\left\|D^{2} u\right\|_{L_{p}\left(\mathbb{R}^{d}\right)} \leq N\|L u-\lambda u\|_{L_{p}\left(\mathbb{R}^{d}\right)},
$$

provided that $\lambda \geq \lambda_{0}$, where $\lambda_{0} \geq 0$ and $N$ depend only on $d, \delta, p, K$, and $R_{0}$.

ii) For any $\lambda>\lambda_{0}$ and $f \in L_{p}\left(\mathbb{R}^{d}\right)$, there exists a unique solution $u \in W_{p}^{2}\left(\mathbb{R}^{d}\right)$ of equation (2.4) in $\mathbb{R}^{d}$.

Proof. We use the idea that solutions to elliptic equations can be viewed as steady state solutions to parabolic equations. By the method of continuity, it suffices to verify the $a$ priori estimate. Let $\lambda_{0}$ be the constant in Theorem 2.2. Take $\lambda \geq \lambda_{0}$. Let $\eta$ be a smooth function on $\mathbb{R}$ supported on $[-2,2]$, and let $\eta(t)=1$ on $[-1,1]$. For a fixed $T>0$, denote $v(t, x)=u(x) \eta(t / T)$. Then $v \in W_{p}^{1,2}$ satisfies

$$
(P-\lambda) v(t, x)=-T^{-1} \eta^{\prime}(t / T) u(x)+\eta(t / T)(L-\lambda) u(x) .
$$

From Theorem 2.2, we have

$$
\lambda\|v\|_{L_{p}}+\sqrt{\lambda}\|D v\|_{L_{p}}+\left\|D^{2} v\right\|_{L_{p}} \leq\left\|\eta(t / T) f-T^{-1} \eta^{\prime}(t / T) u\right\|_{L_{p}} .
$$

Combined with the triangle inequality, this gives

$$
\begin{aligned}
& \|\eta(t / T)\|_{L_{p}(\mathbb{R})}\left(\lambda\|u\|_{L_{p}\left(\mathbb{R}^{d}\right)}+\sqrt{\lambda}\|D u\|_{L_{p}\left(\mathbb{R}^{d}\right)}+\left\|D^{2} u\right\|_{L_{p}\left(\mathbb{R}^{d}\right)}\right) \\
& \quad \leq N\|\eta(t / T)\|_{L_{p}(\mathbb{R})}\|f\|_{L_{p}\left(\mathbb{R}^{d}\right)}+N T^{-1}\left\|\eta^{\prime}(t / T)\right\|_{L_{p}\left(\mathbb{R}_{T}\right)}\|u\|_{L_{p}\left(\mathbb{R}^{d}\right)} .
\end{aligned}
$$

Dividing both sides by $\|\eta(t / T)\|_{L_{p}(\mathbb{R})}$ and letting $T \rightarrow \infty$ yield (2.5). The theorem is proved. 
The theorems above generalize several previously known results on nondivergence form equations with discontinuous coefficients to a large extent. In particular, one can get the solvability of equations to which the results in [14] and [15] are not applicable. We refer the reader to an interesting example given in the end of the Introduction in [19]. Here we give another example for $d=2$.

In the polar coordinates $(\rho, \theta)$, consider the union of two graphs $\rho=e^{-\theta / \varepsilon}$ and $\rho=$ $e^{-(\theta+\pi) / \varepsilon}$ for some $\varepsilon>0$. This curve divides the plane into two connected components. It becomes flat if $\varepsilon$ is small. Let $a^{i j}$ be different constants in these two components. If $\varepsilon=\varepsilon(\gamma)$ is sufficiently small, it is easy to check that the $a^{i j}$ satisfy Assumption 2.4 $(\gamma)$. But there exists no common diffeomorphism $\psi$ that works for all small balls centered at the origin. Therefore, the result in [14 is not applicable in this case even if one uses a partition of the unity.

Remark 2.6. In 9, the $W_{p}^{2}$-solvability was obtained for equations with leading coefficients measurable in two spatial variables and VMO in the others, when $p>2$ is sufficiently close to 2. An interesting problem is whether that result can be extended to equations with variably partially VMO coefficients.

\section{§3. New Estimates FOR NONDiVERGEnCE FORM EQUATIONS}

First, we recall the following embedding type result and a parabolic version of Poincaré's inequality (see, for example, [18] or [20]).

Lemma 3.1. Let $q \geq 1$, and let

$$
\frac{1}{q}<\frac{1}{d+2}+\frac{1}{p}
$$

Then there is a constant $N=N(d, p, q, r, R)$ such that for any $u \in W_{q, \text { loc }}^{1,2}$ and $0<r<$ $R<\infty$ we have

$$
\|u\|_{L_{p}\left(Q_{r}\right)}+\|D u\|_{L_{p}\left(Q_{r}\right)} \leq N\|u\|_{W_{q}^{1,2}\left(Q_{R}\right)} .
$$

Lemma 3.2 (Lemma 5.4 [18]). Let $p \in[1, \infty)$. Then there is a constant $N=N(d, p)$ such that for any $r \in(0, \infty)$ and $u \in C_{\mathrm{loc}}^{\infty}\left(\mathbb{R}^{d+1}\right)$ we have

$$
\begin{array}{r}
\int_{Q_{r}}\left|D u(t, x)-(D u)_{Q_{r}}\right|^{p} d x d t \leq N r^{p} \int_{Q_{r}}\left(\left|D^{2} u(t, x)\right|^{p}+\left|u_{t}\right|^{p}\right) d x d t \\
\int_{Q_{r}}\left|u(t, x)-(u)_{Q_{r}}-x^{j}\left(D_{j} u\right)_{Q_{r}}\right|^{p} d x d t \leq N r^{2 p} \int_{Q_{r}}\left(\left|D^{2} u(t, x)\right|^{p}+\left|u_{t}\right|^{p}\right) d x d t .
\end{array}
$$

Let

$$
P_{0} u=-u_{t}+a^{i j} D_{i j} u, \quad \widetilde{P}_{0} u=-u_{t}+D_{i}\left(a^{i j} D_{j} u\right),
$$

where $a^{i j}=a^{i j}\left(x^{1}\right)$. The proposition below is a special case of Corollary 5.5 in [7].

Proposition 3.3 (Corollary 5.5 in [7]). Suppose $p \in(1, \infty), u \in C_{0}^{\infty}$, and $f, g \in L_{p}$. Then there exists a constant $N>0$, depending only on $d, p$ and $\delta$, such that we have

$$
\lambda\|u\|_{L_{p}}+\sqrt{\lambda}\|D u\|_{L_{p}} \leq N \sqrt{\lambda}\|g\|_{L_{p}}+N\|f\|_{L_{p}},
$$

provided that $\lambda \geq 0$ and $\widetilde{P}_{0} u-\lambda u=f+\operatorname{div} g$. In particular, if $\lambda=0$ and $f=0$, then

$$
\|D u\|_{L_{p}} \leq N\|g\|_{L_{p}}
$$

Our proof relies on the following solvability theorem. 
Theorem 3.4. Let $p \in(1, \infty)$ and $T \in(-\infty, \infty]$. Then for any $u \in W_{p}^{1,2}\left(\mathbb{R}_{T}^{d+1}\right)$ and $\lambda \geq 0$, we have

$$
\begin{array}{r}
\lambda\|u\|_{L_{p}\left(\mathbb{R}_{T}^{d+1}\right)}+\sqrt{\lambda}\|D u\|_{L_{p}\left(\mathbb{R}_{T}^{d+1}\right)}+\left\|D^{2} u\right\|_{L_{p}\left(\mathbb{R}_{T}^{d+1}\right)}+\left\|u_{t}\right\|_{L_{p}\left(\mathbb{R}_{T}^{d+1}\right)} \\
\leq N\left\|P_{0} u-\lambda u\right\|_{L_{p}\left(\mathbb{R}_{T}^{d+1}\right)},
\end{array}
$$

where $N=N(d, p, \delta)>0$. Moreover, for any $f \in L_{p}\left(\mathbb{R}_{T}^{d+1}\right)$ and $\lambda>0$ there is a unique $u \in W_{p}^{1,2}\left(\mathbb{R}_{T}^{d+1}\right)$ solving $P_{0} u-\lambda u=f$ in $\mathbb{R}_{T}^{d+1}$.

Proof. First we assume $T=\infty$. By the method of continuity, it suffices to prove the $a$ priori estimate (3.1) for $u \in C_{0}^{\infty}$. Let

$$
f=P_{0} u-\lambda u \text {. }
$$

The idea is to use the estimate for the corresponding divergence form operator. We make a change of variables:

$$
y^{1}=\varphi\left(x^{1}\right):=\int_{0}^{x^{1}} \frac{1}{a^{11}(s)} d s, \quad y^{j}=x^{j}, \quad j \geq 2 .
$$

It is easily seen that $\varphi$ is a bi-Lipschitz function and

$$
\delta \leq y^{1} / x^{1} \leq \delta^{-1}, \quad D_{y^{1}}=a^{11}\left(x^{1}\right) D_{x^{1}} .
$$

Denote

$$
\begin{aligned}
v\left(t, y^{1}, y^{\prime}\right) & =u\left(t, \varphi^{-1}\left(y^{1}\right), y^{\prime}\right), \quad \tilde{a}^{i j}\left(y^{1}\right)=a^{i j}\left(\varphi^{-1}\left(y^{1}\right)\right), \\
\widetilde{f}(t, y) & =f\left(t, \varphi^{-1}\left(y^{1}\right), y^{\prime}\right) .
\end{aligned}
$$

We define a divergence form operator $\widetilde{P}_{0}$ by

$$
\widetilde{P}_{0} v=-v_{t}+D_{1}\left(\frac{1}{\widetilde{a}^{11}} D_{1} v\right)+\sum_{j=2}^{d} D_{j}\left(\frac{\widetilde{a}^{1 j}+\widetilde{a}^{j 1}}{\widetilde{a}^{11}} D_{1} v\right)+\sum_{i, j=2}^{d} D_{j}\left(\widetilde{a}^{i j} D_{i} v\right) .
$$

Clearly, $v$ satisfies

$$
\widetilde{P}_{0} v-\lambda v=\tilde{f}
$$

in $\mathbb{R}^{d+1}$, and $\widetilde{P}_{0}$ is uniformly nondegenerate. By Proposition 3.3 , we have

$$
\lambda\|v\|_{L_{p}}+\sqrt{\lambda}\|D v\|_{L_{p}} \leq N\|\tilde{f}\|_{L_{p}} .
$$

Therefore,

$$
\lambda\|u\|_{L_{p}}+\sqrt{\lambda}\|D u\|_{L_{p}} \leq N\|f\|_{L_{p}} .
$$

Next we estimate $D^{2} u$. Notice that, for each $k=2, \ldots, d, D_{k} v$ satisfies

$$
\widetilde{P}_{0}\left(D_{k} v\right)-\lambda D_{k} v=D_{k} \tilde{f} .
$$

Again by using Proposition 3.3, we get

$$
\left\|D_{y y^{k}} v\right\|_{L_{p}} \leq N\|\tilde{f}\|_{L_{p}}
$$

which implies

$$
\left\|D_{x x^{\prime}} u\right\|_{L_{p}} \leq N\|f\|_{L_{p}} .
$$

Finally, to estimate $D_{1}^{2} u$, we return to the equation in the original coordinates. From (3.2), we see that $w:=D_{1} u$ satisfies

$$
-w_{t}+D_{1}\left(a^{11} D_{1} w\right)+\Delta_{d-1} w-\lambda w=D_{1} f+\sum_{i j>1} D_{1}\left(\left(\delta_{i j}-a^{i j}\right) D_{i j} u\right) .
$$


We use Proposition 3.3 again to get

$$
\left\|D_{1}^{2} u\right\|_{L_{p}} \leq\|D w\|_{L_{p}} \leq N\|f\|_{L_{p}}+N \sum_{i j>1}\left\|D_{i j} u\right\|_{L_{p}} .
$$

Combining (3.3), (3.4), and (3.5) yields (3.1) by bearing in mind that

$$
u_{t}=a^{i j} D_{i j} u-\lambda u-f .
$$

For general $T \in(-\infty, \infty]$, we use the fact that $u=w$ for $t<T$, where $w \in W_{p}^{1,2}$ solves

$$
P_{0} w-\lambda w=\chi_{t<T}\left(P_{0} u-\lambda u\right)
$$

The theorem is proved.

As a direct consequence of Theorem 3.4, arguing as after Theorem 2.3, we obtain the following.

Corollary 3.5. Let $p \in(1, \infty)$ and $T \in(0, \infty)$. Then for any $f \in L_{p}\left((0, T) \times \mathbb{R}^{d}\right)$ and $\lambda \geq 0$ there is a unique $u \in W_{p}^{1,2}\left((0, T) \times \mathbb{R}^{d}\right)$ solving $P_{0} u-\lambda u=f$ in $(0, T) \times \mathbb{R}^{d}$ and $u(0, \cdot)=0$. Moreover, we have

$$
\begin{array}{r}
\lambda\|u\|_{L_{p}\left((0, T) \times \mathbb{R}^{d}\right)}+\sqrt{\bar{\lambda}}\|D u\|_{L_{p}\left((0, T) \times \mathbb{R}^{d}\right)}+\left\|D^{2} u\right\|_{L_{p}\left((0, T) \times \mathbb{R}^{d}\right)}+\left\|u_{t}\right\|_{L_{p}\left((0, T) \times \mathbb{R}^{d}\right)} \\
\leq N\left\|P_{0} u-\lambda u\right\|_{L_{p}\left((0, T) \times \mathbb{R}^{d}\right)},
\end{array}
$$

where $N=N(d, p, \delta)>0$.

Corollary 3.6. Let $p \in(1, \infty)$ and $u \in W_{p, \text { loc }}^{1,2}$. Then for any $\left(t_{0}, x_{0}\right) \in \mathbb{R}^{d+1}$ and $0<r<R<\infty$,

$$
\begin{aligned}
& \left\|u_{t}\right\|_{L_{p}\left(Q_{r}\left(t_{0}, x_{0}\right)\right)}+\left\|D^{2} u\right\|_{L_{p}\left(Q_{r}\left(t_{0}, x_{0}\right)\right)} \\
& \quad \leq N\left(\left\|P_{0} u\right\|_{L_{p}\left(Q_{R}\left(t_{0}, x_{0}\right)\right)}+\|u\|_{L_{p}\left(Q_{R}\left(t_{0}, x_{0}\right)\right)}+\|D u\|_{L_{p}\left(Q_{R}\left(t_{0}, x_{0}\right)\right)}\right),
\end{aligned}
$$

where $N=N(d, \delta, r, R)$.

Proof. It suffices to apply Theorem 3.4 to $u \eta$, where $\eta$ is a suitable smooth cutoff function.

Now we state and prove the following useful theorem, which gives a Hölder estimate of $D_{x x^{\prime}} u$ for 'harmonic' $u$. This is analogous to Lemma 4.8 in [14, which was used crucially in [19. However, it turns out that the proof of Lemma 4.8 in [14] (see also the proof of Lemma 4.7 in [15]) only works when $p=2$ and employs the special structure of scalar equations. Instead, here we use a bootstrap argument which works for any $p \in(1, \infty)$. This approach also makes it possible to extend the results to systems and higher order equations.

Theorem 3.7. Let $p \in(1, \infty)$ and $u \in C_{0}^{\infty}$. Assume $P_{0} u=0$ in $Q_{2}\left(t_{0}, x_{0}\right)$ for some $\left(t_{0}, x_{0}\right) \in \mathbb{R}^{d+1}$. Then for any $\alpha \in(0,1)$, we have

$$
\left\|D_{x x^{\prime}} u\right\|_{C^{\alpha / 2, \alpha}\left(Q_{1}\left(t_{0}, x_{0}\right)\right)} \leq N\left(\|u\|_{L_{p}\left(Q_{2}\left(t_{0}, x_{0}\right)\right)}+\|D u\|_{L_{p}\left(Q_{2}\left(t_{0}, x_{0}\right)\right)}\right)
$$

for some constant $N=N(d, \delta, \alpha, p)$.

Proof. Without loss of generality, we may assume $\left(t_{0}, x_{0}\right)=(0,0)$. We prove the lemma by a bootstrap argument. Take an increasing sequence $p_{j} \in(1, \infty), j=0,1, \ldots, m$, where $m$ depends only on $d$ and $\alpha$, such that

$$
p_{0}=p, \quad p_{m}>\frac{d+2}{1-\alpha}, \quad \frac{1}{p_{j+1}}<\frac{1}{p_{j}}-\frac{1}{d+2} .
$$


Also, we take a sequence of shrinking cylinders

$$
Q^{(j)}, \quad j=0,1,2, \ldots, 3 m+2,
$$

such that $Q^{(0)}=Q_{2}$ and $Q^{(3 m+2)}=Q_{1}$.

By Corollary 3.6, we have

$$
\|u\|_{W_{p_{0}}^{1,2}\left(Q^{(1)}\right)} \leq N\left(\|u\|_{L_{p}\left(Q^{(0)}\right)}+\|D u\|_{L_{p}\left(Q^{(0)}\right)}\right):=N I .
$$

Then from Lemma 3.1 it follows that

$$
\|u\|_{L_{p_{1}}\left(Q^{(2)}\right)}+\|D u\|_{L_{p_{1}}\left(Q^{(2)}\right)} \leq N\|u\|_{W_{p_{0}}^{1,2}\left(Q^{(1)}\right)} \leq N I .
$$

Since $D_{x^{\prime}} u$ satisfies the same equation in $Q_{2}$, by (3.8) and (3.7) we have

$$
\left\|D_{x x^{\prime}} u\right\|_{L_{p_{1}}\left(Q^{(3)}\right)} \leq N\left(\left\|D_{x^{\prime}} u\right\|_{L_{p_{0}}\left(Q^{(1)}\right)}+\left\|D_{x x^{\prime}} u\right\|_{L_{p_{0}}\left(Q^{(1)}\right)}\right) \leq N I .
$$

Now we write

$$
-u_{t}+a^{11} D_{11} u+\Delta_{d-1} u=\Delta_{d-1} u-\sum_{i j>1} a^{i j} D_{i j} u
$$

and use Corollary 3.6 with $p_{1}$ in place of $p$. Together with (3.8) and (3.9), this gives

$$
\|u\|_{W_{p_{1}}^{1,2}\left(Q^{(4)}\right)} \leq N\left(\|u\|_{L_{p_{1}}\left(Q^{(3)}\right)}+\|D u\|_{L_{p_{1}}\left(Q^{(3)}\right)}+\left\|D_{x x^{\prime}} u\right\|_{L_{p_{1}}\left(Q^{(3)}\right)}\right) \leq N I .
$$

We can iterate (3.8)-(3.10) along with the increasing sequence $p_{j}$ and shrinking cylinders $Q^{(j)}$. After $m$ steps, we reach

$$
\|u\|_{W_{p m}^{1,2}\left(Q^{(3 m+1)}\right)} \leq N I .
$$

Again, since $D_{x^{\prime}} u$ satisfies the same equation in $Q_{2}$, we have

$$
\left\|D_{x^{\prime}} u\right\|_{W_{W_{m}}^{1,2}\left(Q^{(3 m+2)}\right)} \leq N\left(\left\|D_{x^{\prime}} u\right\|_{L_{p}\left(Q^{(1)}\right)}+\left\|D_{x x^{\prime}} u\right\|_{L_{p}\left(Q^{(1)}\right)}\right) \leq N I .
$$

Finally, due to the classical Sobolev embedding theorem of parabolic type, (3.11) gives

$$
\left\|D_{x x^{\prime}} u\right\|_{C^{\alpha / 2, \alpha}\left(Q_{1}\right)} \leq N I \text {. }
$$

The theorem is proved.

Corollary 3.8. Let $p \in(1, \infty)$ and $u \in C_{0}^{\infty}$. Suppose $P_{0} u=0$ in $Q_{2}\left(t_{0}, x_{0}\right)$ for some $\left(t_{0}, x_{0}\right) \in \mathbb{R}^{d+1}$. Then for any $\alpha \in(0,1)$, we have

$$
\left[D_{x x^{\prime}} u\right]_{C^{\alpha / 2, \alpha}\left(Q_{1}\left(t_{0}, x_{0}\right)\right)} \leq N\left(\left\|u_{t}\right\|_{L_{p}\left(Q_{2}\left(t_{0}, x_{0}\right)\right)}+\left\|D^{2} u\right\|_{L_{p}\left(Q_{2}\left(t_{0}, x_{0}\right)\right)}\right)
$$

for some constant $N=N(d, \delta, \alpha, p)$.

Proof. Again we assume $\left(t_{0}, x_{0}\right)=(0,0)$. We notice that $v:=u-(u)_{Q_{2}}-x^{j}\left(D_{j} u\right)_{Q_{2}}$ satisfies the same equation as $u$ in $Q_{2}$. Therefore, by (3.6),

$$
\begin{aligned}
{\left[D_{x x^{\prime}} u\right]_{C^{\alpha / 2, \alpha}\left(Q_{1}\right)} } & =\left[D_{x x^{\prime}} v\right]_{C^{\alpha / 2, \alpha}\left(Q_{1}\right)} \\
& \leq N\left\|u-(u)_{Q_{2}}-x^{j}\left(D_{j} u\right)_{Q_{2}}\right\|_{L_{p}\left(Q_{2}\right)}+N\left\|D u-(D u)_{Q_{2}}\right\|_{L_{p}\left(Q_{2}\right)} .
\end{aligned}
$$

By Lemma 3.2 we see that the right-hand side of (3.13) is less than the right-hand side of (3.12). The corollary is proved.

Corollary 3.9. Suppose $p \in(1, \infty), \kappa \geq 2, r \in(0, \infty)$, and $u \in C_{0}^{\infty}$. Assume that $P_{0} u=0$ in $Q_{\kappa r}\left(t_{0}, x_{0}\right)$ for some $\left(t_{0}, x_{0}\right) \in \mathbb{R}^{d+1}$. Then for any $\alpha \in(0,1)$, we have

$$
\left(\left|D_{x x^{\prime}} u-\left(D_{x x^{\prime}} u\right)_{Q_{r}\left(t_{0}, x_{0}\right)}\right|^{p}\right)_{Q_{r}\left(t_{0}, x_{0}\right)} \leq N \kappa^{-\alpha p}\left(\left|u_{t}\right|^{p}+\left|D^{2} u\right|^{p}\right)_{Q_{\kappa r}\left(t_{0}, x_{0}\right)}
$$

for some constant $N=N(d, \delta, \alpha, p)$. 
Proof. We may assume $\left(t_{0}, x_{0}\right)=(0,0)$. By a scaling argument, it suffices to consider the case where $r=2 / \kappa \leq 1$. By Corollary 3.8, we get

$$
\begin{aligned}
& \left(\left|D_{x x^{\prime}} u-\left(D_{x x^{\prime}} u\right)_{Q_{2 / \kappa}}\right|^{p}\right)_{Q_{2 / \kappa}} \leq N \kappa^{-p \alpha}\left[D_{x x^{\prime}} u\right]_{C^{\alpha / 2, \alpha}\left(Q_{2 / \kappa}\right)}^{p} \\
& \leq N \kappa^{-p \alpha}\left[D_{x x^{\prime}} u\right]_{C^{\alpha / 2, \alpha}\left(Q_{1}\right)}^{p} \leq N \kappa^{-p \alpha}\left(\left|u_{t}\right|^{p}+\left|D^{2} u\right|^{p}\right)_{Q_{2}} .
\end{aligned}
$$

We have a similar estimate for $u_{t}$.

Lemma 3.10. Suppose $p \in(1, \infty), \kappa \geq 2$, and $r>0$. Assume that $u \in C_{0}^{\infty}$ and $P_{0} u=0$ in $Q_{\kappa r}\left(t_{0}, x_{0}\right)$. Then there exist constants $N=N(d, p, \delta)$ and $\alpha=\alpha(d, p, \delta) \in(0,1]$ such that

$$
\left(\left|u_{t}-\left(u_{t}\right)_{Q_{r}\left(t_{0}, x_{0}\right)}\right|^{p}\right)_{Q_{r}\left(t_{0}, x_{0}\right)} \leq N \kappa^{-p \alpha}\left(\left|u_{t}\right|^{p}\right)_{Q_{\kappa r}\left(t_{0}, x_{0}\right)} .
$$

Proof. We may assume $\left(t_{0}, x_{0}\right)=(0,0)$. By scaling, we reduce the general situation to that where $r=1$. Since $L u_{t}=0$ in $Q_{\kappa r}$, by the classical results [16, Lemma 4.2.4] and [21, Theorem 7.21],

$$
\underset{Q_{1 / \kappa}}{\operatorname{Osc}} u_{t} \leq N \kappa^{-\alpha}\left\|u_{t}\right\|_{L_{p}\left(Q_{1}\right)}
$$

with $\alpha$ and $N$ as in the statement. Scaling this estimate shows that

$$
\underset{Q_{1}}{\operatorname{Osc}} u_{t} \leq N \kappa^{-\alpha}\left(\left|u_{t}\right|^{p}\right)_{Q_{\kappa}}^{1 / p} .
$$

It only remains to observe that

$$
\left(\left|u_{t}-\left(u_{t}\right)_{Q_{1}}\right|^{p}\right)_{Q_{1}} \leq N\left(\underset{Q_{1}}{\operatorname{osc}} u_{t}\right)^{p} .
$$

The lemma is proved.

Proposition 3.11. Suppose $p \in(1, \infty), \kappa \geq 4, r>0$, and $u \in C_{0}^{\infty}$. Let $\alpha$ be the constant in Lemma 3.10. Then for any $\left(t_{0}, x_{0}\right) \in \mathbb{R}^{d+1}$, we have

$$
\begin{array}{r}
\left(\left|D_{x x^{\prime}} u-\left(D_{x x^{\prime}} u\right)_{Q_{r}\left(t_{0}, x_{0}\right)}\right|^{p}\right)_{Q_{r}\left(t_{0}, x_{0}\right)}+\left(\left|u_{t}-\left(u_{t}\right)_{Q_{r}\left(t_{0}, x_{0}\right)}\right|^{p}\right)_{Q_{r}\left(t_{0}, x_{0}\right)} \\
\leq N \kappa^{d+2}\left(\left|P_{0} u\right|^{p}\right)_{Q_{\kappa r}\left(t_{0}, x_{0}\right)}+N \kappa^{-\alpha p}\left(\left|u_{t}\right|^{p}+\left|D^{2} u\right|^{p}\right)_{Q_{\kappa r}\left(t_{0}, x_{0}\right)}
\end{array}
$$

for some constant $N=N(d, \delta, p)$.

Proof. The proposition follows from Corollary 3.5, Corollary 3.9. and Lemma 3.10 by decomposing $u$ into a 'harmonic' part and a 'nonharmonic' part; see, for instance, the proof of Proposition 5.3 below or the proof of Theorem 4.5 in 9 .

We finish this section by recalling a generalized version of the Fefferman-Stein theorem. To state this theorem, let

$$
\mathbb{C}_{n}=\left\{C_{n}\left(i_{0}, i_{1}, \ldots, i_{d}\right), i_{0}, \ldots, i_{d} \in \mathbb{Z}\right\}, \quad n \in \mathbb{Z},
$$

be the filtration of partitions given by parabolic dyadic cubes, where

$C_{n}\left(i_{0}, i_{1}, \ldots, i_{d}\right)=\left[i_{0} 2^{-2 n},\left(i_{0}+1\right) 2^{-2 n}\right) \times\left[i_{1} 2^{-n},\left(i_{1}+1\right) 2^{-n}\right) \times \cdots \times\left[i_{d} 2^{-n},\left(i_{d}+1\right) 2^{-n}\right)$.

Theorem 3.12 (Theorem 2.7 in [19]). Let $p \in(1, \infty), U, V, H \in L_{1}$. Assume that $V \geq|U|, H \geq 0$, and that for any $n \in \mathbb{Z}$ and $C \in \mathbb{C}_{n}$ there exists a measurable function $U^{C}$ on $C$ such that $|U| \leq U^{C} \leq V$ on $C$ and

$$
\int_{C}\left|U^{C}-\left(U^{C}\right)_{C}\right| d x d t \leq \int_{C} H d x d t
$$

Then we have

provided that $H, V \in L_{p}$.

$$
\|U\|_{L_{p}}^{p} \leq N\|H\|_{L_{p}}\|V\|_{L_{p}}^{p-1}
$$




\section{§4. Proof of Theorem 2.2}

With the preparations in the previous section, we complete the proof of Theorem 2.2 by adapting the idea in [19].

Lemma 4.1. Suppose $p \in(1, \infty), \hat{a} \in \mathcal{A}$, and $\psi \in \Psi$. Let $\alpha$ be the constant in Lemma 3.10. Denote

$$
\widehat{P} u(t, x)=-u_{t}(t, x)+\widehat{a}^{k l}\left(y^{1}\right) D_{y^{k}} \phi^{i}(y) D_{y^{l}} \phi^{j}(y) D_{i j} u(t, x),
$$

where $y=\psi(x)$ and $\phi=\psi^{-1}$. Then there exist constants $N=N(d, p, \delta)$ and $\nu=$ $\nu(d, \delta) \geq 1$ such that, for any $\kappa \geq 4, r>0$, and $u \in C_{0}^{\infty}$, we have

$$
\begin{aligned}
& \sum_{i j>1}\left(\left|u_{i j}-\left(u_{i j}\right)_{Q_{r}}\right|^{p}\right)_{Q_{r}}+\left(\left|u_{t}-\left(u_{t}\right)_{Q_{r}}\right|^{p}\right)_{Q_{r}} \\
& \quad \leq N \kappa^{d+2}\left(|\widehat{P} u|^{p}+|D u|^{p}\right)_{Q_{\nu \kappa r}}+N \kappa^{-\alpha p}\left(\left|u_{t}\right|^{p}+\left|D^{2} u\right|^{p}\right)_{Q_{\nu \kappa r}},
\end{aligned}
$$

where

$$
u_{i j}(t, x)=\left(D_{y^{i} y^{j}} v\right)(t, \psi(x)), \quad v(t, y)=u(t, \phi(y)) .
$$

Proof. We deduce the lemma from Proposition 3.11 by following the proof of Lemma 3.2 in [19] with some modifications. For the sake of completeness, we give the details. By a translation of the coordinates, we may assume that $\phi(0)=\psi(0)=0$. We define $f=\widehat{P} u$ and $\widehat{P}_{y} v=-v_{t}+\widehat{a}^{k l}\left(y_{1}\right) D_{y^{k} y^{l}} v(t, y)$. Note that

$$
\widehat{P}_{y} v(t, y)=f(t, \phi(y))+\widehat{a}^{k l} D_{y^{k} y^{l}} \phi^{i}\left(D_{x^{i}} u\right)(t, \phi(y)) .
$$

By applying Proposition 3.11 to $\widehat{P}_{y}$, for any $\rho>0$, we get

$$
\begin{aligned}
& \left(\left|D_{y y^{\prime}} v-\left(D_{y y^{\prime}} v\right)_{Q_{\rho}}\right|^{p}\right)_{Q_{\rho}}+\left(\left|v_{t}-\left(v_{t}\right)_{Q_{\rho}}\right|^{p}\right)_{Q_{\rho}} \\
& \leq N \kappa^{d+2}\left(\left|\widehat{P}_{y} v\right|^{p}\right)_{Q_{\kappa \rho}}+N \kappa^{-\alpha p}\left(\left|v_{t}\right|^{p}+\left|D_{y}^{2} v\right|^{p}\right)_{Q_{\kappa \rho}} .
\end{aligned}
$$

Bearing in mind that the scales in $x$ and $y$ coordinates are comparable, we can find constants $N$ and $\nu$, which depend only on $d$ and $\delta$, such that for any measurable function $g$ we have

$$
\begin{gathered}
f_{Q_{\rho}}|g(t, x)| d x d t \leq N f_{Q_{\sqrt{\nu} \rho}}|g(t, \phi(y))| d y d t, \\
f_{Q_{\rho}}|g(t, \phi(y))| d y d t \leq N f_{Q_{\sqrt{\nu} \rho}}|g(t, x)| d x d t .
\end{gathered}
$$

This and the triangle inequality imply

$$
\begin{aligned}
f_{Q_{r}} & \left|u_{i j}-\left(u_{i j}\right)_{Q_{r}}\right|^{p} d x d t \\
& \leq f_{Q_{r}} f_{Q_{r}}\left|u_{i j}\left(t_{1}, x_{1}\right)-u_{i j}\left(t_{2}, x_{2}\right)\right|^{p} d x_{1} d x_{2} d t_{1} d t_{2} \\
& \leq N f_{Q_{\sqrt{\nu} r}} f_{Q_{\sqrt{\nu} r}}\left|D_{y^{i} y^{j}} v\left(t_{1}, y_{1}\right)-D_{y^{i} y^{j}} v\left(t_{2}, y_{2}\right)\right|^{p} d y_{1} d y_{2} d t_{1} d t_{2} \\
& \leq N f_{Q_{\sqrt{\nu} r}}\left|D_{y^{i} y^{j}} v(t, y)-\left(D_{y^{i} y^{j}} v\right)_{\sqrt{\nu} r}\right|^{p} d y d t
\end{aligned}
$$

Similarly,

$$
f_{Q_{r}}\left|u_{t}-\left(u_{t}\right)_{B_{r}}\right|^{p} d x d t \leq N f_{Q_{\sqrt{\nu} r}}\left|v_{t}(t, y)-\left(v_{t}\right)_{Q_{\sqrt{\nu} r}}\right|^{p} d y d t
$$


Moreover, for $y=\psi(x)$ we obviously have

$$
\left|D_{y}^{2} v(y)\right| \leq N\left|D_{x}^{2} u(x)\right|+N\left|D_{x} u(x)\right|, \quad\left|\widehat{P}_{y} v(y)\right| \leq|\widehat{P} u(x)|+N\left|D_{x} u(x)\right| .
$$

By combining the estimates above, we easily deduce (4.1) from (4.3) .

The next result can be viewed as an extension of Lemma 4.1.

Proposition 4.2. Let $p \in(1, \infty), \gamma>0$, and let $\tau, \sigma \in(1, \infty)$ satisfy $1 / \tau+1 / \sigma=1$. Let $\alpha$ be the constant in Lemma 3.10 and $\nu=\nu(d, \delta)>1$ the constant in Lemma 4.1. Assume $b^{i}=c=0$ and $u \in C_{0}^{\infty}$. Then, under Assumption 2.1] $(\gamma)$, for any $r \in(0, \infty)$ and $\left(t_{0}, x_{0}\right) \in \mathbb{R}^{d+1}$ there exists a diffeomorphism $\psi \in \Psi$ and a positive constant $N=N(d, p, \delta, \tau)$ such that for any $\kappa \geq 4$,

$$
\begin{aligned}
\sum_{i j>1}\left(\left|u_{i j}-\left(u_{i j}\right)_{Q_{r}\left(t_{0}, x_{0}\right)}\right|^{p}\right)_{Q_{r}\left(t_{0}, x_{0}\right)}+\left(\left|u_{t}-\left(u_{t}\right)_{Q_{r}\left(t_{0}, x_{0}\right)}\right|^{p}\right)_{Q_{r}\left(t_{0}, x_{0}\right)} \\
\leq N \kappa^{d+2}\left(|P u|^{p}+|D u|^{p}\right)_{Q_{\nu \kappa r}\left(t_{0}, x_{0}\right)}+N \kappa^{d+2} \gamma^{1 / \sigma}\left(\left|D^{2} u\right|^{p \tau}\right)_{Q_{\nu \kappa r}\left(t_{0}, x_{0}\right)}^{1 / \tau} \\
\quad+N \kappa^{-p \alpha}\left(\left|u_{t}\right|^{p}\right)_{Q_{\nu \kappa r}\left(t_{0}, x_{0}\right)}+N\left(\kappa^{d+2} R^{p}+\kappa^{-p \alpha}\right)\left(\left|D^{2} u\right|^{p}\right)_{Q_{\nu \kappa r}\left(t_{0}, x_{0}\right)},
\end{aligned}
$$

provided that $u$ vanishes outside $Q_{R}$ for some $R \in\left(0, R_{0}\right]$. Here the $u_{i j}$ are defined in (4.2).

Proof. We fix $\kappa \geq 4$ and $r \in(0, \infty)$. Choose $Q$ to be $Q_{\nu \kappa r}\left(t_{0}, x_{0}\right)$ if $\nu \kappa r<R$ and $Q_{R}$ if $\nu \kappa r \geq R$. Let $\left(t^{*}, x^{*}\right)$ be the center of $Q$. By Assumption 2.1 $(\gamma)$ and (2.1), we can find $\psi \in \Psi$ and $\bar{a}=\bar{a}(s) \in \mathcal{A}$ satisfying (2.3). We set

$$
\widehat{a}^{k l}(s)=\bar{a}^{i j}(s)\left(D_{i} \psi^{k}\right)\left(x^{*}\right)\left(D_{j} \psi^{l}\right)\left(x^{*}\right), \quad y^{*}=\psi\left(x^{*}\right) .
$$

By Lemma 4.1 with a shift of the coordinates, for $i j>1$ we have

$$
\begin{aligned}
& \left(\left|u_{i j}-\left(u_{i j}\right)_{Q_{r}\left(t_{0}, x_{0}\right)}\right|^{p}\right)_{Q_{r}\left(t_{0}, x_{0}\right)}+\left(\left|u_{t}-\left(u_{t}\right)_{Q_{r}\left(t_{0}, x_{0}\right)}\right|^{p}\right)_{Q_{r}\left(t_{0}, x_{0}\right)} \\
& \quad \leq N \kappa^{d+2}\left(|\hat{P} u|^{p}+|D u|^{p}\right)_{Q_{\nu \kappa r}\left(t_{0}, x_{0}\right)}+N \kappa^{-\alpha p}\left(\left|u_{t}\right|^{p}+\left|D^{2} u\right|^{p}\right)_{Q_{\nu \kappa r}\left(t_{0}, x_{0}\right)},
\end{aligned}
$$

where $N$ depends only on $d$ and $\delta$. By the definition of $\widehat{P}$,

$$
\int_{Q_{\nu \kappa r}\left(t_{0}, x_{0}\right)}|\widehat{P} u|^{p} d x d t \leq N \int_{Q_{\nu \kappa r}\left(t_{0}, x_{0}\right)}|P u|^{p} d x d t+N I+N J
$$

where

$$
\begin{aligned}
I & :=\int_{Q_{\nu \kappa r}\left(t_{0}, x_{0}\right) \cap Q_{R}}\left|\left(\left(D_{y^{k}} \phi^{i} D_{y^{l}} \phi^{j}\right)(\psi)-\left(D_{y^{k}} \phi^{i} D_{y^{l}} \phi^{j}\right)\left(y^{*}\right)\right) \hat{a}^{k l}\left(\psi^{1}\right) D_{i j} u\right|^{p} d x d t \\
& \leq N\left\|\left(D_{y^{k}} \phi^{i} D_{y^{l}} \phi^{j}\right)(\psi)-\left(D_{y^{k}} \phi^{i} D_{y^{l}} \phi^{j}\right)\left(y^{*}\right)\right\|_{L_{\infty}\left(Q_{R}\right)}^{p} \int_{Q_{\nu \kappa r}\left(t_{0}, x_{0}\right)}\left|D_{i j} u\right|^{p} d x d t \\
& \leq N R^{p} \int_{Q_{\nu \kappa r}\left(t_{0}, x_{0}\right)}\left|D_{i j} u\right|^{p} d x d t
\end{aligned}
$$

and

$$
J:=\int_{Q_{\nu \kappa r}\left(t_{0}, x_{0}\right) \cap Q_{R}}\left|\left(\bar{a}^{i j}\left(\psi^{1}\right)-a^{i j}(t, x)\right) D_{i j} u\right|^{p} d x d t .
$$

We have used (2.2) in (4.7). By Hölder's inequality, we estimate $J$ by

$$
J \leq N J_{1}^{1 / \sigma} J_{2}^{1 / \tau}
$$

where

$$
J_{1}=\sum_{i, j} \int_{Q_{\nu \kappa r}\left(t_{0}, x_{0}\right) \cap Q_{R}}\left|\bar{a}^{i j}\left(\psi^{1}\right)-a^{i j}\right|^{p \sigma} d x d t, \quad J_{2}=\int_{Q_{\nu \kappa r}\left(t_{0}, x_{0}\right)}\left|D^{2} u\right|^{p \tau} d x d t .
$$


By Assumption $2.1(\gamma)$,

$$
J_{1} \leq \sum_{i, j} \int_{Q}\left|\bar{a}^{i j}\left(\psi^{1}\right)-a^{i j}\right|^{p \sigma} d x d t \leq N \gamma|Q| \leq N(\nu \kappa r)^{d+2} \gamma .
$$

Combined with (4.5)-(4.8), this yields (4.4). The theorem is proved.

From Proposition 4.2 we obtain the following corollary by simply using the triangle inequality.

Corollary 4.3. Suppose $q \in(1, \infty), \gamma>0, \kappa \geq 4$, and let $\tau, \sigma \in(1, \infty)$ satisfy $1 / \tau+$ $1 / \sigma=1$. Let $\alpha$ be the constant in Lemma 3.10. Suppose Assumption 2.1] $(\gamma)$ is satisfied. Assume $b^{i}=c=0$. Then for any $n \in \mathbb{Z}$ and $C \in \mathbb{C}_{n}$, there exists a diffeomorphism $\psi \in \Psi$ and a constant $N=N(d, \delta, q, \tau)$ such that, with any $u \in C_{0}^{\infty}$ vanishing outside $Q_{R}$ for some $R \in\left(0, R_{0}\right]$, we have

$$
\left(\left|u_{t}-\left(u_{t}\right)_{C}\right|\right)_{C}+\sum_{i j>1}\left(\left|u_{i j}-\left(u_{i j}\right)_{C}\right|\right)_{C} \leq N(\mathrm{~g})_{C},
$$

where the $u_{i j}(t, x)$ are defined by (4.2) and

$$
\begin{aligned}
\mathrm{g}= & \kappa^{\frac{d+2}{q}}\left(\mathbb{M}\left(|P u|^{q}\right)\right)^{\frac{1}{q}}+\kappa^{\frac{d+2}{q}}\left(\mathbb{M}\left(|D u|^{q}\right)\right)^{\frac{1}{q}}+\kappa^{\frac{d+2}{q}} \gamma^{\frac{1}{\sigma q}}\left(\mathbb{M}\left(\left|D^{2} u\right|^{q \tau}\right)\right)^{\frac{1}{q \tau}} \\
& +\kappa^{-\alpha}\left(\mathbb{M}\left(\left|u_{t}\right|^{q}\right)\right)^{\frac{1}{q}}+\left(\kappa^{\frac{d+2}{q}} R+\kappa^{-\alpha}\right)\left(\mathbb{M}\left(\left|D^{2} u\right|^{q}\right)\right)^{\frac{1}{q}} .
\end{aligned}
$$

Moreover, we have

$$
\left|u_{t}\right|+\left|D^{2} u\right| \leq N \sum_{i j>1}\left|u_{i j}\right|+N\left|u_{t}\right|+N|D u|+N|P u| .
$$

Proposition 4.4. Let $p \in(1, \infty)$. Assume $b^{i}=c=0$. Then there exist positive constants $\gamma, N$ and $R \in(0,1]$ depending only on $d, p$, and $\delta$ such that under Assumption 2.1 $(\gamma)$, for any $u \in C_{0}^{\infty}$ vanishing outside $Q_{R R_{0}}$ we have

$$
\left\|u_{t}\right\|_{L_{p}}+\left\|D^{2} u\right\|_{L_{p}} \leq N\|P u\|_{L_{p}}+N\|D u\|_{L_{p}} .
$$

Proof. Set $f=P u \in C_{0}^{\infty}$. Let $\gamma>0, \kappa \geq 4$, and $R \in(0,1]$ be constants to be specified later. Let $q=(1+p) / 2 \in(1, p)$ and $\tau=2(1+2 p) /(3+3 p)>1$ such that $p>q \tau$. We take $n \in \mathbb{Z}$, and $C \in \mathbb{C}_{n}$, and let $\psi \in \Psi$ be the diffeomorphism occurring in Corollary 4.3. Recall the definition of $u_{i j}$ in (4.2). We set

$$
U=\left|u_{t}\right|+\left|D^{2} u\right|, \quad U^{C}=\left|u_{t}\right|+\sum_{i j>1}\left|u_{i j}\right|+|D u|+|f|, \quad V=\left|u_{t}\right|+\left|D^{2} u\right|+|D u|+|f| .
$$

From (4.10), we have $U \leq N U^{C}$. By using the triangle inequality and (4.9),

$$
\begin{aligned}
\left(\left|U^{C}-\left(U^{C}\right)_{C}\right|\right)_{C} \leq & 2\left(\left|u_{t}-\left(u_{t}\right)_{C}\right|\right)_{C}+2 \sum_{i j>1}\left(\left|u_{i j}-\left(u_{i j}\right)_{C}\right|\right) \\
& +2\left(\left|D u-(D u)_{C}\right|\right)_{C}+2\left(\left|f-(f)_{C}\right|\right)_{C} \\
\leq & N(\mathrm{~g}+|D u|+|f|)_{C} .
\end{aligned}
$$

Now Theorem 3.12 with $H=\mathrm{g}+|D u|+f$ yields

$$
\left\|u_{t}\right\|_{L_{p}}^{p}+\left\|D^{2} u\right\|_{L_{p}}^{p} \leq N\|U\|_{L_{p}}^{p} \leq N\|H\|_{L_{p}}\|V\|_{L_{p}}^{p-1} \leq N(\varepsilon)\|H\|_{L_{p}}^{p}+\varepsilon\|V\|_{L_{p}}^{p} .
$$

By taking a small $\varepsilon>0$, it follows that

$$
\left\|u_{t}\right\|_{L_{p}}^{p}+\left\|D^{2} u\right\|_{L_{p}}^{p} \leq N\|\mathrm{~g}\|_{L_{p}}^{p}+N\|D u\|_{L_{p}}^{p}+N\|f\|_{L_{p}}^{p} .
$$


We use the definition of $\mathrm{g}$ and the Hardy-Littlewood maximal function theorem (recall that $p>q \tau>q)$ to deduce the following inequality from (4.12):

$$
\begin{aligned}
\left\|u_{t}\right\|_{L_{p}}+\left\|D^{2} u\right\|_{L_{p}} \leq & N \kappa^{\frac{d+2}{q}}\|P u\|_{L_{p}}+N \kappa^{\frac{d+2}{q}}\|D u\|_{L_{p}}+N \kappa^{-\alpha}\left\|u_{t}\right\|_{L_{p}} \\
& +N\left(\kappa^{\frac{d+2}{q}} \gamma^{\frac{1}{\sigma q}}+\kappa^{\frac{d+2}{q}} R R_{0}+\kappa^{-\alpha}\right)\left\|D^{2} u\right\|_{L_{p}} .
\end{aligned}
$$

By choosing $\kappa$ sufficiently large, and then taking $\gamma$ and $R$ in (4.13) sufficiently small and such that

$$
N\left(\kappa^{\frac{d+2}{q}} \gamma^{\frac{1}{\sigma q}}+\kappa^{\frac{d+2}{q}} R+\kappa^{-\alpha}\right) \leq 1 / 2,
$$

we come to (4.11). The theorem is proved.

Proof of Theorem 2.2. For $T=\infty$, the theorem follows in a by now standard way from Proposition 4.4 by using a partition of unity and an idea by S. Agmon. For general $T \in(-\infty,+\infty]$, we again use the argument at the end of the proof of Theorem 3.4.

\section{§5. SoboleV SPACES With MIXED NORMS}

In this section we consider parabolic equations in Sobolev spaces with mixed norms in the spirit of [18. As pointed out in [18, the interest in results concerning equations in spaces with mixed Sobolev norms arises, for example, when one wants to get better regularity of traces of solutions for each time slide (see, for instance, [23, 24] and the references therein).

Our objective is to prove the following theorem, which generalizes Theorem 2.2.

Theorem 5.1. For any $1<p \leq q<\infty$ there exists $\gamma=\gamma(d, \delta, p, q)>0$ such that, under Assumption $2.1(\gamma)$, for any $T \in(-\infty,+\infty]$ the following is true.

i) For any $u \in W_{q, p}^{1,2}\left(\mathbb{R}_{T}^{d+1}\right)$,

$$
\begin{array}{r}
\lambda\|u\|_{L_{q, p}\left(\mathbb{R}_{T}^{d+1}\right)}+\sqrt{\lambda}\|D u\|_{L_{q, p}\left(\mathbb{R}_{T}^{d+1}\right)}+\left\|D^{2} u\right\|_{L_{q, p}\left(\mathbb{R}_{T}^{d+1}\right)}+\left\|u_{t}\right\|_{L_{q, p}\left(\mathbb{R}_{T}^{d+1}\right)} \\
\leq N\|P u-\lambda u\|_{L_{q, p}\left(\mathbb{R}_{T}^{d+1}\right)},
\end{array}
$$

provided that $\lambda \geq \lambda_{0}$, where $\lambda_{0} \geq 0$ and $N$ depend only on $d, \delta, p, q, K$, and $R_{0}$.

ii) For any $\lambda>\lambda_{0}$ and $f \in L_{q, p}\left(\mathbb{R}_{T}^{d+1}\right)$, there exists a unique solution $u \in W_{q, p}^{1,2}\left(\mathbb{R}_{T}^{d+1}\right)$ of equation (1.1) in $\mathbb{R}_{T}^{d+1}$.

Since the case where $p=q$ was covered in Theorem 2.2 in the sequel we assume $p<q$. We make a few preparations before the proof.

Lemma 5.2. Let $p, q \in(1, \infty), b=c=0$. Then there exists a constant $\gamma_{0}=$ $\gamma_{0}(d, p, q, \delta)>0$ such that, under Assumption $2.1\left(\gamma_{0}\right)$, for any $r \in\left(0, R_{0}\right]$ and $u \in W_{q, \text { loc }}^{1,2}$ satisfying $P u=0$ in $Q_{2 r}$ we have $D^{2} u \in L_{p}\left(Q_{r}\right)$ and

$$
\left(\left|D^{2} u\right|^{p}\right)_{Q_{r}}^{1 / p} \leq N\left(\left|D^{2} u\right|^{q}\right)_{Q_{2 r}}^{1 / q},
$$

where $N$ depends only on $d, p, q$, and $\delta$.

Proof. First we assume $R_{0}=1$. In this case, the lemma is proved in Corollary 6.4 of [18] with the only difference that the coefficients $a^{i j}$ are assumed to be in $\mathrm{VMO}_{x}$ in that paper. The proof of that in [18] employs the $L_{p}$-solvability of equations with $\mathrm{VMO}_{x}$ coefficients. Since the solvability is already established with coefficients satisfying Assumption [2.1 $\left(\gamma_{0}\right)$ with $\gamma_{0}$ depending on $d, p, q$, and $\delta$, we can just reproduce the proof with almost no change.

For general $R_{0} \in(0,1]$, we make a change of variables $(t, x) \rightarrow\left(R_{0}^{2} t, R_{0} x\right)$ and notice that the new coefficients satisfy Assumption 2.1 $\left(\gamma_{0}\right)$ with $R_{0}$ replaced by 1 . The lemma is proved. 
The next proposition improves Proposition 4.2 .

Proposition 5.3. Let $p \in(1, \infty)$ and $\gamma>0$. Let $\alpha$ be the constant in Lemma 3.10. $\nu=\nu(d, \delta)>1$ the constant in Lemma 4.1, and $\gamma_{0}=\gamma_{0}(d, p, 2 p, \delta)$ the constants in Lemma [5.2. Assume $b^{i}=c=0$ and $u \in C_{0}^{\infty}$. Then, under Assumption $2.1(\gamma)$ with $\gamma \in\left(0, \gamma_{0}\right]$, the following is true. For any $R \in\left(0, R_{0}\right], \kappa \geq 16, r \in\left(0, R \kappa^{-1} \nu^{-1}\right]$, and $\left(t_{0}, x_{0}\right) \in \mathbb{R}^{d+1}$, there exists a diffeomorphism $\psi \in \Psi$ such that

$$
\begin{gathered}
\sum_{i j>1}\left(\left|u_{i j}-\left(u_{i j}\right)_{Q_{r}\left(t_{0}, x_{0}\right)}\right|^{p}\right)_{Q_{r}\left(t_{0}, x_{0}\right)}+\left(\left|u_{t}-\left(u_{t}\right)_{Q_{r}\left(t_{0}, x_{0}\right)}\right|^{p}\right)_{Q_{r}\left(t_{0}, x_{0}\right)} \\
\leq N \kappa^{d+2}\left(|P u|^{p}+|D u|^{p}\right)_{Q_{\nu \kappa r}\left(t_{0}, x_{0}\right)}+N \kappa^{-p \alpha}\left(\left|u_{t}\right|^{p}\right)_{Q_{\nu \kappa r}\left(t_{0}, x_{0}\right)} \\
+N\left(\kappa^{d+2} \gamma^{1 / 2}+\kappa^{d+2} R^{p}+\kappa^{-p \alpha}\right)\left(\left|D^{2} u\right|^{p}\right)_{Q_{\nu \kappa r}\left(t_{0}, x_{0}\right)},
\end{gathered}
$$

where the $u_{i j}$ are defined in (4.2) and $N=N(d, p, \delta)>0$.

Proof. Without loss of generality, we assume that $\left(t_{0}, x_{0}\right)=(0,0)$. We may also assume that the $a^{i j}$ are infinitely differentiable by using standard mollifications if necessary.

Let $f:=P u$. Take a cutoff function $\eta \in C_{0}^{\infty}$ such that $\eta=1$ on $Q_{\nu \kappa r / 2}$ and $\eta=0$ outside the closure of $Q_{\nu \kappa r} \cup\left(-Q_{\nu \kappa r}\right)$. By Theorem 2.3. there exists a unique solution $w \in W_{p}^{1,2}\left((-1,0) \times \mathbb{R}^{d}\right)$ of

$$
P w=f \eta \text { on }(-1,0) \times \mathbb{R}^{d}, \quad w(-1, \cdot)=0 .
$$

By the classical theory, we have $w \in C^{\infty}\left((-1,0) \times \mathbb{R}^{d}\right)$. Let $h=u-w$; this function is also smooth and satisfies

$$
P h=f(1-\eta) \text { on }(-1,0) \times \mathbb{R}^{d}, \quad P h=0 \text { on } Q_{\nu \kappa r / 2} .
$$

First, we estimate $h$. Choose $Q$ to be $Q_{\nu \kappa r / 4}$. By Assumption $2.1(\gamma)$ and (2.1), we can find $\psi \in \Psi$ and $\bar{a}=\bar{a}(s) \in \mathcal{A}$ satisfying (2.3). By repeating the proof of Proposition 4.2 with $h$ in place of $u$ (recall $\kappa / 4 \geq 4$ ), we get

$$
\begin{aligned}
\sum_{i j>1}\left(\left|h_{i j}-\left(h_{i j}\right)_{Q_{r}}\right|^{p}\right)_{Q_{r}}+\left(\left|h_{t}-\left(h_{t}\right)_{Q_{r}}\right|^{p}\right)_{Q_{r}} \\
\leq N \kappa^{d+2}\left(|D h|^{p}\right)_{Q_{\nu \kappa r / 4}}+N \kappa^{d+2} \gamma^{1 / 2}\left(\left|D^{2} h\right|^{2 p}\right)_{Q_{\nu \kappa r / 4}}^{1 / 2} \\
\quad+N \kappa^{-p \alpha}\left(\left|h_{t}\right|^{p}\right)_{Q_{\nu \kappa r / 4}}+N\left(\kappa^{d+2} R^{p}+\kappa^{-p \alpha}\right)\left(\left|D^{2} h\right|^{p}\right)_{Q_{\nu \kappa r / 4}} \\
\leq N \kappa^{d+2}\left(|D h|^{p}\right)_{Q_{\nu \kappa r}}+N \kappa^{d+2} \gamma^{1 / 2}\left(\left|D^{2} h\right|^{p}\right)_{Q_{\nu \kappa r}} \\
\quad+N \kappa^{-p \alpha}\left(\left|h_{t}\right|^{p}\right)_{Q_{\nu \kappa r}}+N\left(\kappa^{d+2} R^{p}+\kappa^{-p \alpha}\right)\left(\left|D^{2} h\right|^{p}\right)_{Q_{\nu \kappa r}}
\end{aligned}
$$

where the $h_{i j}$ are defined in the same way as $u_{i j}$. In the last inequality we used the relation $P h=0$ on $Q_{\nu \kappa r / 2}$ and Lemma 5.2.

Next we estimate $w$. By Theorem 2.3 , we have

$$
\|w\|_{W_{p}^{1,2}\left((-1,0) \times \mathbb{R}^{d}\right)} \leq N\|f \eta\|_{L_{p}\left((-1,0) \times \mathbb{R}^{d}\right)} \leq N\|f\|_{L_{p}\left(Q_{\nu \kappa r}\right)} .
$$

Therefore,

$$
\begin{aligned}
& \left(\left|w_{t}\right|^{p}+|D w|^{p}+\left|D^{2} w\right|^{p}\right)_{Q_{r}} \leq N \kappa^{d+2}\left(|f|^{p}\right)_{Q_{\nu \kappa r}} \\
& \left(\left|w_{t}\right|^{p}+|D w|^{p}+\left|D^{2} w\right|^{p}\right)_{Q_{\nu \kappa r}} \leq N\left(|f|^{p}\right)_{Q_{\nu \kappa r}}
\end{aligned}
$$


Since $\left|w_{i j}\right| \leq N|D w|+N\left|D^{2} w\right|$, combining (5.2), (5.3) and (5.4) and using the triangle inequality, we get

$$
\begin{aligned}
\sum_{i j>1}\left(\left|u_{i j}-\left(u_{i j}\right)_{Q_{r}}\right|^{p}\right)_{Q_{r}}+\left(\left|u_{t}-\left(u_{t}\right)_{Q_{r}}\right|^{p}\right)_{Q_{r}} \\
\leq N \sum_{i j>1}\left(\left|h_{i j}-\left(h_{i j}\right)_{Q_{r}}\right|^{p}\right)_{Q_{r}}+N\left(\left|h_{t}-\left(h_{t}\right)_{Q_{r}}\right|^{p}\right)_{Q_{r}} \\
\quad+N\left(\left|w_{t}\right|^{p}+|D w|^{p}+\left|D^{2} w\right|^{p}\right)_{Q_{r}} \\
\leq N \kappa^{d+2}\left(|D h|^{p}\right)_{Q_{\nu \kappa r}}+N\left(\kappa^{d+2} \gamma^{1 / 2}+\kappa^{d+2} R^{p}+\kappa^{-p \alpha}\right)\left(\left|D^{2} h\right|^{p}\right)_{Q_{\nu \kappa r}} \\
\quad+N \kappa^{-p \alpha}\left(\left|h_{t}\right|^{p}\right)_{Q_{\nu \kappa r}}+N \kappa^{d+2}\left(|f|^{p}\right)_{Q_{\nu \kappa r}} \\
\leq N \kappa^{d+2}\left(|D u|^{p}\right)_{Q_{\nu \kappa r}}+N\left(\kappa^{d+2} \gamma^{1 / 2}+\kappa^{d+2} R^{p}+\kappa^{-p \alpha}\right)\left(\left|D^{2} u\right|^{p}\right)_{Q_{\nu \kappa r}} \\
\quad+N \kappa^{-p \alpha}\left(\left|u_{t}\right|^{p}\right)_{Q_{\nu \kappa r}}+N \kappa^{d+2}\left(|f|^{p}\right)_{Q_{\nu \kappa r}},
\end{aligned}
$$

which is exactly the right-hand side of (5.1). The theorem is proved.

The next corollary is deduced from Proposition 5.3 .

Corollary 5.4. Under the assumptions of Proposition [5.3, for any $R \in\left(0, R_{0}\right], \kappa \geq 16$ and any interval $[S, T)$ such that $(T-S)^{1 / 2}=: r \in\left(0, R \kappa^{-1} \nu^{-1}\right]$, we have

$$
\begin{aligned}
f_{(S, T)} & f_{(S, T)}|\varphi(t)-\varphi(s)|^{p} d t d s \\
\leq & N \kappa^{d+2} f_{\left(T-(\nu \kappa r)^{2}, T\right)} \zeta(t)^{p} d t \\
& +N\left(\kappa^{d+2} R^{p}+\kappa^{-p \alpha}+\kappa^{d+2} \gamma^{1 / 2}\right) f_{\left(T-(\nu \kappa r)^{2}, T\right)} \rho(t)^{p} d t
\end{aligned}
$$

where $N=N(d, p, \delta)>0$, and

$$
\begin{aligned}
\zeta(t)^{p} & =\int_{\mathbb{R}^{d}}|P u(t, x)|^{p}+|D u(t, x)|^{p} d x \\
\rho(t)^{p} & =\int_{\mathbb{R}^{d}}\left|D^{2} u(t, \cdot)\right|^{p}+\left|u_{t}(t, \cdot)\right|^{p} d x, \\
\varphi(t)^{p} & =\int_{\mathbb{R}^{d}} f_{B_{r}(y)}\left|u_{t}(t, \cdot)\right|^{p}+\sum_{i j>1}\left|u_{i j}^{(y)}(t, \cdot)\right|^{p} d x d y .
\end{aligned}
$$

Here for each $y \in \mathbb{R}^{d}$, the $u_{i j}^{(y)}$ are defined in (4.2) with $Q=Q_{\nu \kappa r / 4}(T, y)$ and $\psi$ in Assumption 2.1 .

Proof. By the triangle inequality, the left-hand side of (5.5) is less than a constant $N$ times

$$
\begin{aligned}
& f_{(S, T)} f_{(S, T)} \int_{\mathbb{R}^{d}} f_{B_{r}(y)}\left|u_{t}(t, \cdot)-u_{t}(s, \cdot)\right|^{p}+\sum_{i j>1}\left|u_{i j}^{(y)}(t, \cdot)-u_{i j}^{(y)}(s, \cdot)\right|^{p} d x d y d t d s \\
& \leq N \int_{\mathbb{R}^{d}} \sum_{i j>1}\left(\left|u_{i j}^{(y)}-\left(u_{i j}^{(y)}\right)_{Q_{r}(T, y) \mid}\right|^{p}\right)_{Q_{r}(T, y)}+\left(\left|u_{t}-\left(u_{t}\right)_{Q_{r}(T, y)}\right|^{p}\right)_{Q_{r}(T, y)} d y .
\end{aligned}
$$

By Proposition 5.3, the last expression is less than $N$ times

$\int_{\mathbb{R}^{d}} \kappa^{d+2}\left(|P u|^{p}+|D u|^{p}\right)_{Q_{\nu \kappa r}(T, y)}+\left(\kappa^{d+2}\left(R^{p}+\gamma^{\frac{1}{2}}\right)+\kappa^{-p \alpha}\right)\left(\left|D^{2} u\right|^{p}+\left|u_{t}\right|^{p}\right)_{Q_{\nu \kappa r}(T, y)} d y$, which is less than the right-hand side of (5.5). 
Finally, we prove the following estimate, which implies Theorem 5.1 in the same way as Proposition 4.4 implies Theorem 2.2

Proposition 5.5. Let $1<p<q<\infty$. Assume $b^{i}=c=0$. Then there exist positive constants $\gamma, N$, and $R \in(0,1]$ depending only on $d, p, q$, and $\delta$ such that under Assumption $2.1(\gamma)$, for any $u \in C_{0}^{\infty}$ vanishing outside $\left(-R^{4} R_{0}^{2}, 0\right) \times \mathbb{R}^{d}$, we have

$$
\left\|u_{t}\right\|_{L_{q, p}}+\left\|D^{2} u\right\|_{L_{q, p}} \leq N\|P u\|_{L_{q, p}}+N\|D u\|_{L_{q, p}} .
$$

Proof. Set $f=P u$. Recall the definitions of $\varphi, \rho$, and $\zeta$ in Corollary [5.4. Let $\gamma \in\left(0, \gamma_{0}\right]$, $\kappa \in[16, \infty)$, and $R \in(0,1]$ be numbers to be chosen later, where $\gamma_{0}=\gamma_{0}(d, p, 2 p, \delta)$ is taken from Lemma 5.2. Assume $u$ vanishes outside $\left(-R^{4} R_{0}^{2}, 0\right) \times \mathbb{R}^{d}$. Denote $f=P u$ and

$$
\begin{aligned}
\mathrm{g}=\mathrm{g}(t)= & \left(\kappa^{\frac{d+2}{p}} R_{0} R+\kappa^{-\alpha}+\kappa^{\frac{d+2}{p}} \gamma^{\frac{1}{2 p}}+(R \kappa)^{2\left(1-\frac{1}{p}\right)}\right)\left(\mathbb{M}\left(\rho^{p}\right)\right)^{\frac{1}{p}} \\
& +N\left(\kappa^{\frac{d+2}{p}}+(R \kappa)^{2\left(1-\frac{1}{p}\right)}\right)\left(\mathbb{M}\left(\zeta^{p}\right)\right)^{\frac{1}{p}} .
\end{aligned}
$$

Let $\mathbb{C}_{n}$ be the filtration of partitions given by the dyadic intervals

$$
\left\{\left[j 2^{-2 n},(j+1) 2^{-2 n}\right), j \in \mathbb{Z}\right\} .
$$

For any $C=[S, T) \in \mathbb{C}_{n}$, we set

$$
\begin{aligned}
& U(t)=\left\|u_{t}(t, \cdot)\right\|_{L_{p}\left(\mathbb{R}^{d}\right)}+\left\|D^{2} u(t, \cdot)\right\|_{L_{p}\left(\mathbb{R}^{d}\right)}, \\
& V(t)=\left\|u_{t}(t, \cdot)\right\|_{L_{p}\left(\mathbb{R}^{d}\right)}+\left\|D^{2} u(t, \cdot)\right\|_{L_{p}\left(\mathbb{R}^{d}\right)}+\|D u(t, \cdot)\|_{L_{p}\left(\mathbb{R}^{d}\right)}+\|f(t, \cdot)\|_{L_{p}\left(\mathbb{R}^{d}\right)},
\end{aligned}
$$

and

$$
U^{C}(t)= \begin{cases}\varphi(t)+\|D u(t, \cdot)\|_{L_{p}\left(\mathbb{R}^{d}\right)}+\|f(t, \cdot)\|_{L_{p}\left(\mathbb{R}^{d}\right)} & \text { if } 2^{-n} \leq R R_{0} \kappa^{-1} \nu^{-1} \\ V(t) & \text { otherwise. }\end{cases}
$$

It is easily seen that $U \leq N U^{C} \leq N V$ in $C$. We claim

$$
\left(U^{C}-\left(U^{C}\right)_{C}\right)_{C} \leq N\left(\mathrm{~g}+\|D u(t, \cdot)\|_{L_{p}\left(\mathbb{R}^{d}\right)}+\|f(t, \cdot)\|_{L_{p}\left(\mathbb{R}^{d}\right)}\right)_{C} \cdot
$$

Indeed, if $2^{-n} \leq R_{0} R \kappa^{-1} \nu^{-1}$, by a shift of the origin we get (5.7) from Corollary 5.4. Otherwise, we have

$$
\begin{aligned}
\left(U^{C}-\left(U^{C}\right)_{C}\right)_{C} & \leq 2 f_{(S, T)} \chi_{\left(-R_{0}^{2} R^{4}, 0\right)}|V(t)| d t \\
& \leq 2\left(f_{(S, T)} \chi_{\left(-R_{0}^{2} R^{4}, 0\right)} d t\right)^{1-1 / p}\left(f_{(S, T)}|V(t)|^{p} d t\right)^{1 / p} \\
& \leq N(R \kappa)^{2(1-1 / p)}\left(\mathbb{M}\left(\rho^{p}+\zeta^{p}\right)\left(t_{0}\right)\right)^{1 / p}
\end{aligned}
$$

for any $t_{0} \in C$. This proves the claim.

Now by Theorem 3.12 with $d=0, q$ in place of $p$ and

$$
H(t):=\mathrm{g}(t)+\|D u(t, \cdot)\|_{L_{p}\left(\mathbb{R}^{d}\right)}+\|f(t, \cdot)\|_{L_{p}\left(\mathbb{R}^{d}\right)},
$$

we get

$$
\left\|u_{t}\right\|_{L_{q, p}}^{q}+\left\|D^{2} u\right\|_{L_{q, p}}^{q} \leq N\|U\|_{L_{q}}^{q} \leq N\|H\|_{L_{q}}\|V\|_{L_{q}}^{q-1} \leq N(\varepsilon)\|H\|_{L_{q}}^{q}+\varepsilon\|V\|_{L_{q}}^{q} .
$$

Taking a small $\varepsilon>0$, we see that

$$
\left\|u_{t}\right\|_{L_{q, p}}^{q}+\left\|D^{2} u\right\|_{L_{q, p}}^{q} \leq N\|\mathrm{~g}\|_{L_{q}}^{q}+N\|D u\|_{L_{q, p}}^{q}+N\|f\|_{L_{q, p}}^{q} .
$$


We use the definition of $\mathrm{g}$ and the Hardy-Littlewood maximal function theorem (recall $p<q$ ) to deduce from (5.8) the estimate

$$
\begin{aligned}
\left\|u_{t}\right\|_{L_{q, p}} & +\left\|D^{2} u\right\|_{L_{q, p}} \leq N\left(\kappa^{\frac{d+2}{p}}+(R \kappa)^{2\left(1-\frac{1}{p}\right)}\right)\left(\|f\|_{L_{q, p}}+\|D u\|_{L_{q, p}}\right) \\
& +N\left(\kappa^{\frac{d+2}{p}} R_{0} R+\kappa^{-\alpha}+\kappa^{\frac{d+2}{p}} \gamma^{\frac{1}{2 p}}+(R \kappa)^{2\left(1-\frac{1}{p}\right)}\right)\left(\left\|u_{t}\right\|_{L_{q, p}}+\left\|D^{2} u\right\|_{L_{q, p}}\right) .
\end{aligned}
$$

By choosing $\kappa$ sufficiently large, and then $\gamma$ and $R$ in (5.9) sufficiently small and such that

$$
N\left(\kappa^{\frac{d+2}{p}} R+\kappa^{-\alpha}+\kappa^{\frac{d+2}{p}} \gamma^{\frac{1}{2 p}}+(R \kappa)^{2\left(1-\frac{1}{p}\right)}\right) \leq 1 / 2
$$

we come to (5.6). The theorem is proved.

\section{ACKNOWLEDGMENT}

The author is sincerely grateful to Nicolaŭ V. Krylov for very helpful comments on the first draft of the article. The author also would like to thank Alexander Nazarov for his careful reading and useful remarks.

\section{REFERENCES}

[1] E. Acerbi and G. Mingione, Gradient estimates for a class of parabolic systems, Duke Math. J. 136 (2007), no. 2, 285-320. MR2286632 (2007k:35211)

[2] S. Byun and L. Wang, $L^{p}$-estimates for general nonlinear elliptic equations, Indiana Univ. Math. J. 56 (2007), no. 6, 3193-3221. MR2375715 (2008m:35131)

[3] S. Agmon, A. Douglis, and L. Nirenberg, Estimates near the boundary for solutions of elliptic partial differential equations satisfying general boundary conditions. I, Comm. Pure Appl. Math. 12 (1959), 623-727; II , ibid., 17 (1964), 35-92. MR0125307 (23:A2610) MR0162050(28:5252)

[4] M. Bramanti and M. Cerutti, $W_{p}^{1,2}$ solvability for the Cauchy-Dirichlet problem for parabolic equations with VMO coefficients, Comm. Partial Differential Equations 18 (1993), no. 9-10, 1735-1763. MR $1239929(94 \mathrm{j}: 35180)$

[5] F. Chiarenza, M. Frasca, and P. Longo, Interior $W^{2, p}$ estimates for nondivergence elliptic equations with discontinuous coefficients, Ricerche Mat. 40 (1991), no. 1, 149-168. MR.1191890 (93k:35051)

[6] _,$W^{2, p}$-solvability of the Dirichlet problem for nondivergence elliptic equations with VMO coefficients, Trans. Amer. Math. Soc. 336 (1993), no. 2, 841-853. MR1088476 (93f:35232)

[7] H. Dong, Solvability of parabolic equations in divergence form with partially BMO coefficients, J. Funct. Anal. 258 (2010), no. 7, 2145-2172. MR2584743 (2011b:35187)

[8] H. Dong and D. Kim, Elliptic equations in divergence form with partially BMO coefficients, Arch. Rational Mech. Anal. 196 (2010), no. 1, 25-70. MR2601069(2011b:35086)

[9] H. Dong and N. V. Krylov, Second-order elliptic and parabolic equations with $B\left(\mathbb{R}^{2}\right.$, VMO) coefficients, Trans. Amer. Math. Soc. 362 (2010), no. 12, 6477-6494. MR2678983

[10] G. Di Fazio, $L^{p}$ estimates for divergence form elliptic equations with discontinuous coefficients, Boll. Un. Mat. Ital. A (7) 10 (1996), no. 2, 409-420. MR.1405255 (97e:35034)

[11] D. Kim, Parabolic equations with measurable coefficients. II, J. Math. Anal. Appl. 334 (2007), no. 1, 534-548. MR2332574(2008f:35160)

[12] _ Elliptic and parabolic equations with measurable coefficients in $L_{p}$-spaces with mixed norms, Methods Appl. Anal. 15 (2008), no. 4, 437-467. MR2550072 (2010j:35566)

[13] _ Parabolic equations with partially BMO coefficients and boundary value problems in Sobolev spaces with mixed norms, Potential Anal. 33 (2010), no. 1, 17-46. MR2644213 (2011h:35120)

[14] D. Kim and N. V. Krylov, Elliptic differential equations with coefficients measurable with respect to one variable and VMO with respect to the others, SIAM J. Math. Anal. 39 (2007), no. 2, 489-506. MR2338417 (2008j:35031)

[15] _ Parabolic equations with measurable coefficients, Potential Anal. 26 (2007), no. 4, 345-361. MR2300337 (2008f:35161)

[16] N. V. Krylov, Nonlinear elliptic and parabolic equations of the second order, Nauka, Moscow, 1985; English transl., Math. Appl. (Soviet Series), vol. 7, D. Reidel Publ. Co., Dordrecht, 1987. MR0815513 (87h:35002), MR0901759 (88d:35005)

[17] - Parabolic and elliptic equations with VMO coefficients, Comm. Partial Differential Equations 32 (2007), no. 1-3, 453-475. MR2304157 (2008a:35125)

[18] _ Parabolic equations with VMO coefficients in Sobolev spaces with mixed norms, J. Funct. Anal. 250 (2007), no. 2, 521-558. MR2352490 (2008f:35164) 
[19] Second-order elliptic equations with variably partially VMO coefficients, J. Funct. Anal. 257 (2009), no. 6, 1695-1712. MR2540989(2010j:35096)

[20] O. A. Ladyzhenskaya, V. A. Solonnikov, and N. N. Ural'tseva, Linear and quasilinear equations of parabolic type, Nauka, Moscow, 1967; English transl., Transl. Math. Monogr., vol. 23, Amer. Math. Soc., Providence, RI, 1967. MR0241822(39:3159b)

[21] G. Lieberman, Second order parabolic differential equations, World Sci. Publ. Co., Inc., River Edge, NJ, 1996. MR 1465184 (98k:35003)

[22] _ A mostly elementary proof of Morrey space estimates for elliptic and parabolic equations with VMO coefficients, J. Funct. Anal. 201 (2003), no. 2, 457-479. MR1986696 (2004b:35025)

[23] L. Softova and P. Weidemaier, Quasilinear parabolic problems in spaces of maximal regularity, J. Nonlinear Convex Anal. 7 (2006), 529-540. MR2287547(2008k:35251)

[24] P. Weidemaier, Maximal regularity for parabolic equations with inhomogeneous boundary conditions in Sobolev spaces with mixed $L_{p}$-norm, Electron. Res. Announc. Amer. Math. Soc. 8 (2002), 47-51. MR 1945779 (2003k:35088)

Division of Applied Mathematics, Brown University, 182 George Street, Providence, Rhode ISLAND 02912

E-mail address: Hongjie_Dong@brown.edu

Received 20/JUN/2010

Originally published in English 